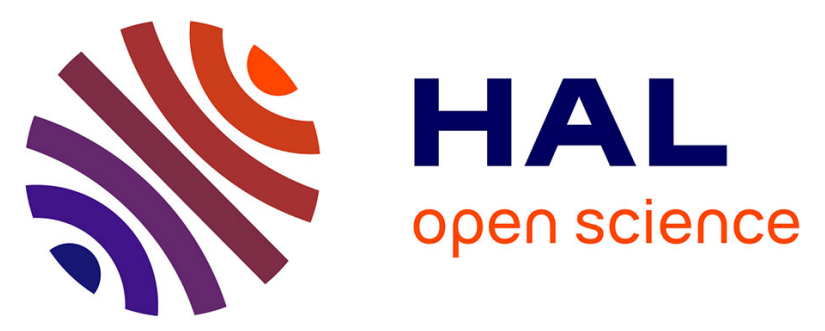

\title{
Influences réciproques des racines et des parties aériennes sur le rendement de l'aubergine (Solanum melongena L.) : résultats de greffages inter-variétaux Marie-Christine Daunay, Philippe Malet, Evelyne Jullian
}

\section{- To cite this version:}

Marie-Christine Daunay, Philippe Malet, Evelyne Jullian. Influences réciproques des racines et des parties aériennes sur le rendement de l'aubergine (Solanum melongena L.): résultats de greffages inter-variétaux. Agronomie, 1986, 6 (3), pp.293-297. hal-00884878

\section{HAL Id: hal-00884878 \\ https://hal.science/hal-00884878}

Submitted on 1 Jan 1986

HAL is a multi-disciplinary open access archive for the deposit and dissemination of scientific research documents, whether they are published or not. The documents may come from teaching and research institutions in France or abroad, or from public or private research centers.
L'archive ouverte pluridisciplinaire HAL, est destinée au dépôt et à la diffusion de documents scientifiques de niveau recherche, publiés ou non, émanant des établissements d'enseignement et de recherche français ou étrangers, des laboratoires publics ou privés. 


\title{
Influences réciproques des racines et des parties aériennes sur le rendement de l'aubergine (Sola- num melongena L.) : résultats de greffages inter-variétaux
}

\author{
Marie-Christine DAUNAY \& Philippe MALET $\left({ }^{*}\right)$ \\ avec la collaboration technique de Evelyne JULLIAN \\ I.N.R.A.. Station d'Amélioration des Plantes marấchères \\ (*) Station de Bioclimatologie. Centre de Recherches d'Avignon, I 84140 Montfatel
}

RÉSUMÉ Afin de mesurer les effets de la croissance racinaire et des parties aériennes sur la production de l'aubergine de
plein champ irriguée, les racines et les parties aériennes d'une variété méditerranéenne ont été échangées par
greffage avec celles d'une variété venant d'Extrême-Orient. Les performances des plantes inter-écotypiques
montrent que les racines et le feuillage ont des influences réciproques et équivalentes sur le nombre de fruits ;
par contre, le développement racinaire a une influence prédominante sur le poids moyen des fruits. Aussi avec
une croissance aérienne et racinaire supérieure, la variété méditerranéenne a le meilleur rendement en culture
irriguée de plein champ dans le Sud de la France.

Mots clés additionnels : rendement, climat, adaptation, écotype.

SUMMARY

Reciprocal effects of roots and shoots on yield of eggplant (Solanum melongena L.) : results from intervarietal grafting.

Measurements have been made of the effects of root growth and shoot development on irrigated eggplant fruit production. Roots and shoots of a Mediterranean variety and an other from the Far East were exchanged by grafting. Growth and fruit production of these inter-ecotype plants (tables $1 \& 2$ ) showed that roots and shoots had an equal and reciprocal influence on fruit number, whereas the root growth effect was mainly on mean fruit weight. Therefore with better root and shoot growth, the Mediterranean variety has the largest fruit production under irrigation in the south of France.

Additional key words : yield, climate, adaptation, ecotype.

\section{INTRODUCTION}

La sélection de l'aubergine est encore de nos jours pratiquée au sein de chaque zone traditionnelle de culture ; aussi les variétés ont-elles des caractéristiques de développement typiques de leur aire d'origine. FILOV (1958) puis, d'une manière plus simplifiée, POCHARD $\&$ SERIEYS (1974) et POCHARD (1979) les ont regroupées selon ces critères en écotypes. Ils distinguent notamment l'écotype méditerranéen (M), de précocité de floraison moyenne, à la végétation assez vigoureuse et peu anthocyanée, originaire d'un climat « chaud et sec », de l'écotype extrême-oriental (EO), très précoce, à la végétation généralement moins touffue et très anthocyanée, originaire d'un climat «chaud et humide $»$.
Parmi les nombreux critères qui distinguent ces 2 écotypes, POCHARD (1979) a observé que, pour de jeunes plantes cultivées en milieu hydroponique, les chevelus racinaires des variétés $M$ étaient plus développés que ceux des variétés EO. Cette différence, également manifeste chez des plantes adultes cultivées en plein champ en Avignon (DAUNAY, 1986), n'est pas liée à la précocité de floraison. Par contre, le gain total en hauteur et le rendement ont tendance à varier dans le même sens que la «vigueur racinaire".

Notre objectif est ici de comparer l'effet « vigueur des racines » à l'effet «vigueur des parties aériennes » sur la fructification. Pour cela, il est nécessaire d'analyser la contribution des racines à la croissance aérienne et à la fructification ainsi que la contribution réciproque des parties aériennes sur la croissance raci- 
naire. Aussi avons-nous associé les vigueurs différentes des écotypes $\mathrm{M}$ et $\mathrm{EO}$ par des greffages intervariétaux, ainsi que Troughton \& WHITTINGTON (1970) recommandent de le faire pour ce type d'étude. Des plantes « inter-écotypiques », avec une partie aérienne appartenant à un écotype différent de celui du système racinaire (EO sur $M$ et $M$ sur $E O$ ), et les plantes témoins autogreffées ( $\mathrm{EO}$ sur $\mathrm{EO}$ et $\mathrm{M}$ sur $\mathrm{M}$ ) ont été mises au champ et leurs performances de croissance et de fructification notées au cours de toute la saison de culture.

Ce protocole était possible parce que l'aubergine se greffe facilement (BEYRIES, 1979) et que son cycle de développement, relativement court (5 à 6 mois dans nos conditions), permet d'obtenir, en un bref délai, des résultats agronomiques complets. Il a été exécuté en 1983 en utilisant une variété représentative de chaque écotype.

\section{MATÉRIEL ET MÉTHODES}

\section{A. Matériel végétal et conduite des essais}

Le greffage a été réalisé entre une variété de l'écotype méditerranéen (M), « LF3-24 » (notée LF3), et une variété de l'écotype extrême oriental (EO), "Liu Yé Qié » (notée LIU). Avec les 2 traitements témoins d'autogreffes, on a ainsi obtenu 4 formules (tabl. 1). Chacune était représentée par 35 plantes : 7 blocs de 5 plantes avec, en bordure des parcelles élémentaires, des plantes de la variété LIU choisie en raison de son faible encombrement végétatif.

Le greffage a été effectué en serre au moment où les plantes avaient entre 7 et 9 feuilles selon la technique du «greffage en tête » (BEYRIES, 1979; PIERRE, 1982), chaque plante fournissant à la fois un portegreffe et un greffon. Dans le cas des autogreffes, on a procédé à un échange de greffons entre plantes. La soudure entre greffon et porte-greffe étant totale au bout de 8 à $10 \mathrm{j}$, la plantation au champ a eu lieu dès que les greffons avaient repris leur croissance soit moins de 3 semaines après le greffage.

\section{B. Variables mesurées et méthodes de mesure}

Sur chaque plante on a mesuré plusieurs fois au cours de l'été la hauteur du rameau le plus long (du sol à l'apex, avec une incertitude de quelques $\mathrm{cm}$ ) et le rendement en fruits (décompte du nombre de fruits et détermination de leur poids au g près). Le jour de l'arrachage de l'essai, des observations sommaires ont été faites sur la morphologie des racines. Après nettoyage et passage à l'étuve à $95^{\circ} \mathrm{C}$ pendant $48 \mathrm{~h}$, le poids sec des racines a été déterminé au cg près.

\section{RÉSULTATS}

\section{A. Croissance en hauteur de la partie aérienne}

L'influence du greffon comme celle du porte-greffe est hautement significative (tabl. 1 \& 2). L'écart entre formules de greffes qui diffèrent seulement par leur système racinaire (fig. 1) montre que les racines de LF3 (écotype M) favorisent la croissance en hauteur alors que les racines de LIU (écotype EO) la réduisent. Il n'y a pas d'interaction greffon $\times$ porte-greffe ni pour la hauteur finale (tabl. 2), ni pour le gain total en hauteur entre la première et la dernière mesure.

\section{B. Production de fruits}

L'influence du greffon et celle du porte-greffe sont hautement significatives pour le rendement total en poids et nombre de fruits par plante (tabl. $1 \& 2$ ). Ces résultats sont illustrés par les figures $2 \& 3$ où l'on voit que les racines de LF3 augmentent le rendement alors que celles de LIU le réduisent. Les porte-greffes ont en outre une influence significative sur le poids moyen.

On a noté que la réduction du poids moyen des fruits de LF3 greffée sur LIU s'accompagne d'un raccourcissement très net de leur longueur par rapport à la normale.

\section{Système racinaire}

\section{Aspect}

Les systèmes racinaires des variétés autogreffées ont les mêmes caractéristiques que celles décrites par DAUNAY (1982) sur plantes non greffées : LIU (de l'écotype $\mathrm{EO}$ ) a des racines peu fournies, peu ramifiées et assez horizontales; au contraire LF3 (de

TABLEAU

Résultats cumulés moyens par plante.

Mean cumulative results per plant.

\begin{tabular}{|c|c|c|c|c|c|c|c|c|c|c|c|}
\hline \multirow{3}{*}{ Greffon } & \multirow{3}{*}{ Porte-greffe } & \multicolumn{4}{|c|}{ Croissance (a) } & \multicolumn{6}{|c|}{ Rendement en fruits } \\
\hline & & \multicolumn{2}{|c|}{ Hauteur } & \multicolumn{2}{|c|}{ Poids sec racines } & \multicolumn{2}{|c|}{ Poids total } & \multicolumn{2}{|c|}{ Nombre total } & \multicolumn{2}{|c|}{ Poids moyen } \\
\hline & & $\mathrm{cm}$ & $* *$ & $\mathrm{~g}$ & $* *$ & $\mathrm{~kg}$ & $* *$ & Nombre & $* *$ & $\mathrm{~g}$ & * \\
\hline \multirow{2}{*}{ LIU } & LIU & 72,3 & $\mathrm{~d}$ & 7,4 & $\mathrm{c}$ & 2,34 & $\mathrm{~b}$ & 9,1 & $\mathrm{c}$ & 258 & $\mathrm{~b}$ \\
\hline & $\mathrm{LF} 3$ & 83,2 & $c$ & 10,7 & $c$ & 3,08 & $b$ & 11,2 & $\mathrm{bc}$ & 274 & $\mathrm{a}$ \\
\hline \multirow{2}{*}{ LF3 } & LIU & 112,5 & $b$ & 17,7 & $\mathrm{~b}$ & 2,81 & b & 11,8 & b & 237 & c \\
\hline & LF3 & 130,6 & $\mathrm{a}$ & 29,0 & $a$ & 4,28 & $\mathrm{a}$ & 16,0 & $a$ & 268 & $a b$ \\
\hline
\end{tabular}


TABLEAU 2

Analyse de variance des résultats cumulés moyens par plante : valeurs des $F$ et seuils de signification : ${ }^{*}=0,05 ;{ }^{* *} P=0,01$. Analysis of variance of the mean cumulative results per plant : $F$ values and significance levels : ${ }^{*} P=0.05 ;{ }^{* *} P=0.01$.

\begin{tabular}{|c|c|c|c|c|c|c|}
\hline \multirow{2}{*}{$\begin{array}{l}\text { Origine de la } \\
\text { variation }\end{array}$} & \multirow[b]{2}{*}{ DDL } & \multicolumn{2}{|c|}{ Croissance (a) } & \multicolumn{3}{|c|}{ Rendement en fruits } \\
\hline & & Hauteur & Poids sec racines & Poids total & Nombre total & Poids moyen \\
\hline Blocs & 6 & 0,92 & 1,57 & 0,62 & 0,55 & 0,35 \\
\hline Traitements & 3 & $182,05 * *$ & $143,06 * *$ & $13,40 * *$ & $13,56 * *$ & $4,25 *$ \\
\hline greffon & 1 & $487,15 * *$ & $317,40 * *$ & $11,84 * *$ & $20,80^{* *}$ & 3,80 \\
\hline porte-greffe & 1 & $55,13 * *$ & $83,74 * *$ & $24,67 * *$ & $17,58 * *$ & $7,87 *$ \\
\hline interaction & 1 & 3,88 & $25,04 * *$ & 3,68 & 2,29 & 1,08 \\
\hline Erreur & 18 & & & & & \\
\hline
\end{tabular}

(a) mesurée le 20 septembre.

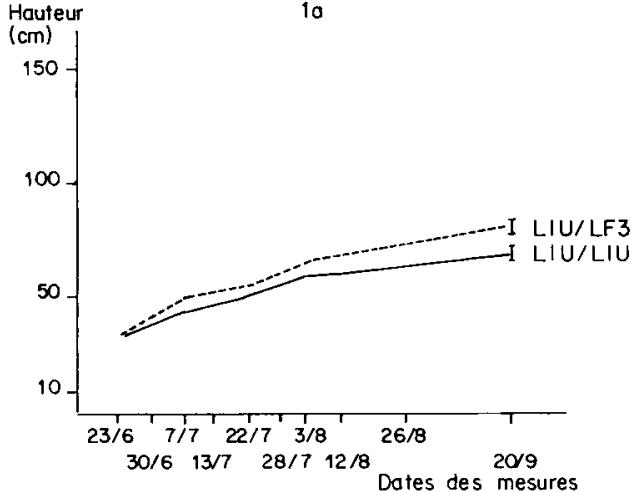

Figure 1

Croissance en hauteur.

1a. Influence des racines de LF3 (écotype $M$ ) sur $L I U$

1a. Influence of $L F 3$ roots ( $M$ ecotype) on $L I U$

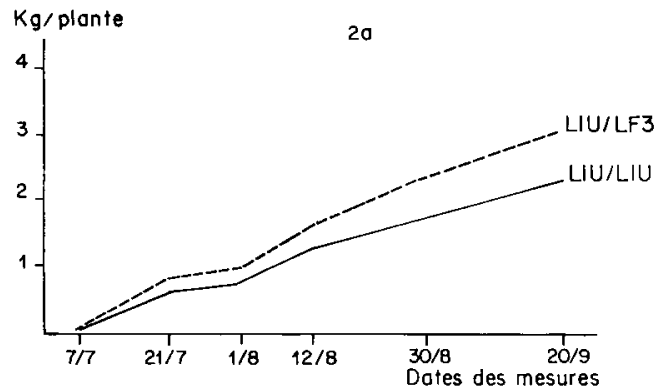

Figure 2

Rendement cumulé en poids de fruits $(\mathrm{kg} / \mathrm{plante})$.

$2 a$. Influence des racines de LF3 (écotype M) sur LIU

2a. Influence of LF3 roots (M ecotype) on LIU.

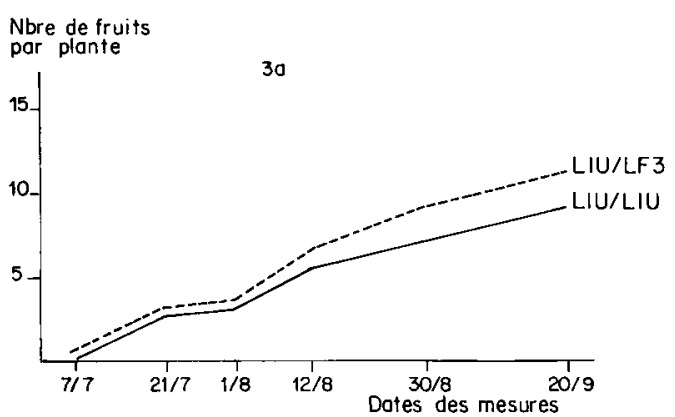

Figure 3

Rendement cumulé en nombre de fruits par plante. $3 a$. Influence des racines de $L F 3$ (écotype $M$ ) sur $L I U$ 3a. Influence of $L F 3$ roots ( $M$ ecotype) on LIU

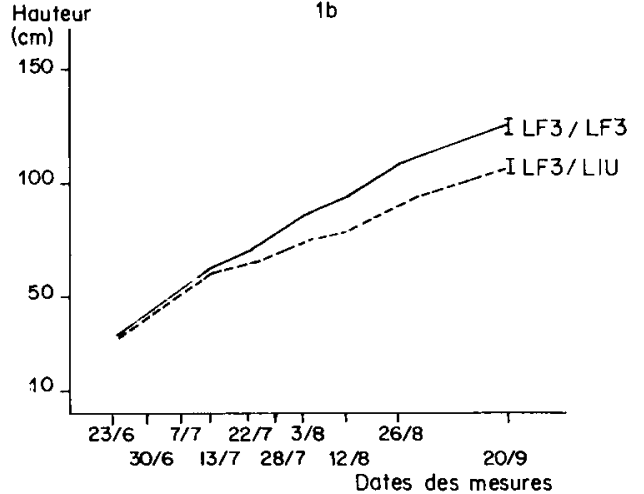

Growth in height.

Ib. Influence des racines de LIU (écotype EO) sur LF3.

1b. Influence of $L I U$ roots (EO ecotype) on LF3.

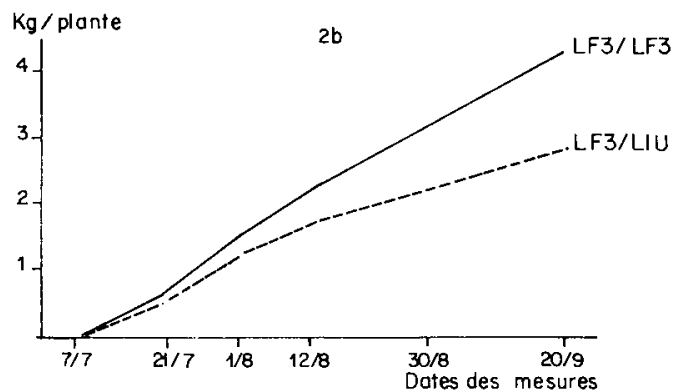

Cumulative weight of fruits $(\mathrm{kg} / \mathrm{plants})$.

$2 b$. Influence des racines de LIU (écotype Eo) sur LF3.

2b. Influence of LIU roots (EO ecotype). on LF3

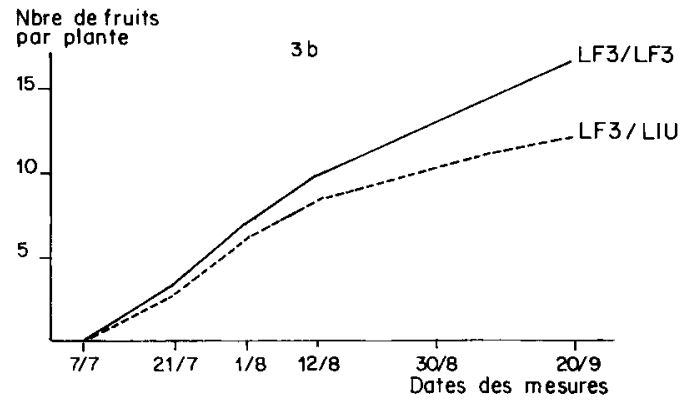

Cumulative fruit number per plant.

3b. Influence des racines de LIU (écotype EO) sur LF3.

3b. Influence of LIU roots (EO ecotype) on LF3. 
l'écotype $\mathrm{M}$ ) a des racines bien développées, très ramifiées et orientées obliquement. Par contre le greffage intervariétal modifie les systèmes racinaires : ceux de LF3 sont réduits (plus faible diamètre, moindre envergure et configuration plus horizontale) par la partie aérienne de LIU. Inversement, les racines de LIU sont "fortifiées » (augmentation du diamètre, plus grande envergure, configuration plus "plongeante " des racines) par la partie aérienne de LF3.

\section{Poids sec}

L'influence du greffon comme celle du porte-greffe sont hautement significatives (tabl. 2) ; il s'y ajoute une interaction greffon $\times$ porte-greffe également significative. L'écart entre formules de greffes différant seulement pour la partie aérienne (tabl. 1) montre que les greffons de LF3 (écotype M) augmentent la croissance racinaire alors que les greffons de LIU (écotype EO) la réduisent.

\section{DISCUSSION}

L'échange par greffage des parties aériennes et racinaires entre une variété extrême-orientale (LIU) et une variété méditerranéenne (LF3) modifie incontestablement à la fois la croissance des parties aériennes et celle des systèmes racinaires. Notons que les plantes se rétablissent parfaitement du choc imposé par le greffage car les performances des variétés autogreffées et leurs classements sont absolument comparables à ceux des variétés non greffées (DAUNAY, 1986).

Le tableau 3 montre qu'un porte-greffe LF3 placé sous un greffon LF3 (dernière ligne) apporte un accroissement général des performances de croissance et de rendement plus important que lorsqu'il est placé sous un greffon LIU ( $7^{\mathrm{e}}$ ligne). Il y a donc un effet dépressif des parties aériennes de LIU sur le potentiel d'amélioration en croissance et en rendement apporté par un porte-greffe LF3. De même, l'accroissement général des performances de croissance et de rendement apporté par un greffon LF3 est plus important en présence d'un porte-greffe LF3 (4e ligne) qu'en présence d'un porte-greffe LIU ( $3^{e}$ ligne). Il y a donc aussi un effet dépressif des racines de LIU sur le potentiel d'amélioration qu'apporte un greffon LF3. Quant à l'interaction greffon $\times$ porte-greffe pour le poids sec des racines (tabl. 2), elle résulte de l'influence bénéfique des greffons et des porte-greffes de LF3, significativement plus forte lorsque l'autre partie de la plante provient encore de LF3.

Le tableau 3 montre aussi que cette influence bénéfique est plus intense sur la croissance en hauteur de la partie aérienne que sur la croissance des fruits, mesurée par leur poids moyen (cf. aussi les différences entre les valeurs des F du tableau 2). Cependant, ce sont sans doute les différences très nettes de hauteur finale entre intergreffes et autogreffes (fig. 1 et tabl. 1) qui entraînent des variations dans le même sens du nombre total de fruits produits. La ramification de l'aubergine est grossièrement dichotomique, aux irrégularités près (DAUNAY, 1982), c'est-à-dire qu'à un étage floral $i$ et un nombre de rameaux de $2^{i}$ correspond un nombre de fleurs (et de fruits potentiels) de $2^{i-1}$. Ainsi, bien que nous ne l'ayons pas mesuré, c'est probablement un changement du nombre de ramifications qui est à l'origine de la relation positive entre la croissance totale en hauteur et le nombre de fruits.

L'effet stimulant des greffons de LF3 engendre un extraordinaire développement racinaire $(+158 \mathrm{p}$. 100) ; quant à celui des porte-greffes de LF3 sur la croissance aérienne $(+48 \mathrm{p}$. 100) et le poids total de fruits $(+43$ p. 100), il n'est pas négligeable non plus. Ceci laisse à penser que le développement racinaire est à l'origine de l' "adaptation » de cette variété aux conditions agroclimatiques méditerranéennes (forte ETP, irrigations espacées et/ou irrégulières). Si l'on confronte nos résultats à ceux d'ABDELHAFEEZ \& CORNILLON (1976) et CORNILLON \& DAUPLE (1981), on peut en déduire que c'est probablement par le maintien d'une alimentation en eau plus régulière et plus abondante que les racines agissent sur les performances aériennes. En effet, ces auteurs avaient obtenu une amélioration de la croissance en hauteur et/ou de la fructification d'aubergines non greffées similaire à celle provoquée par les racines $\mathbf{M}$ dans nos expériences de greffes, en augmentant la fréquence d'apport de l'irrigation (à une dose équilibrant l'ETP).

TABLEAU 3

Effets greffon (en haut) et porte-greffe (en bas) de la variété LF3 sur la croissance et le rendement (en \%). Scion (upper part) and rootstock (lower part) effects of the LF3 variety on growth and yield (\%).

\begin{tabular}{|c|c|c|c|c|c|c|c|c|c|c|c|}
\hline \multirow{3}{*}{$\begin{array}{c}\text { Greffon } \\
\text { LIU }\end{array}$} & \multirow{3}{*}{ 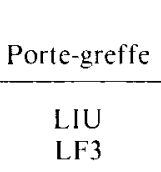 } & \multicolumn{4}{|c|}{ Croissance (a) } & \multicolumn{6}{|c|}{ Rendement en fruits } \\
\hline & & \multicolumn{2}{|c|}{ Hauteur } & \multicolumn{2}{|c|}{ Poids sec racines } & \multicolumn{2}{|c|}{ Poids total } & \multicolumn{2}{|c|}{ Nombre total } & \multirow{2}{*}{$\begin{array}{c}\text { Poids } \\
100 \\
106\end{array}$} & \multirow{2}{*}{$\begin{array}{r}\text { moyen } \\
100\end{array}$} \\
\hline & & $\begin{array}{l}100 \\
115\end{array}$ & 100 & $\begin{array}{l}100 \\
145\end{array}$ & 100 & $\begin{array}{l}100 \\
132\end{array}$ & 100 & $\begin{array}{l}100 \\
123\end{array}$ & 100 & & \\
\hline LF3 & $\begin{array}{l}\text { LIU } \\
\text { LF3 }\end{array}$ & $\begin{array}{l}156 \\
181\end{array}$ & 157 & $\begin{array}{l}239 \\
392\end{array}$ & 258 & $\begin{array}{l}120 \\
183\end{array}$ & 131 & $\begin{array}{l}130 \\
176\end{array}$ & 137 & $\begin{array}{r}92 \\
104\end{array}$ & 95 \\
\hline $\begin{array}{l}\text { LIU } \\
\text { LF3 }\end{array}$ & LIU & $\begin{array}{l}100 \\
156\end{array}$ & 100 & $\begin{array}{l}100 \\
239\end{array}$ & 100 & $\begin{array}{l}100 \\
120\end{array}$ & 100 & $\begin{array}{l}100 \\
130\end{array}$ & 100 & $\begin{array}{r}100 \\
92\end{array}$ & 100 \\
\hline $\begin{array}{l}\text { LIU } \\
\text { LF3 }\end{array}$ & LF3 & $\begin{array}{l}115 \\
181\end{array}$ & 148 & $\begin{array}{l}145 \\
392\end{array}$ & 158 & $\begin{array}{l}132 \\
183\end{array}$ & 143 & $\begin{array}{l}123 \\
176\end{array}$ & 130 & $\begin{array}{l}106 \\
104\end{array}$ & 109 \\
\hline
\end{tabular}

(a) mesurée le 20 septembre. 
Comme parties aériennes et racinaires sont liées par des relations fonctionnelles qui déterminent un ratio d'équilibre pondéral entre elles (KRAMER, 1969 ; BIERHUIZEN, 1974 ; WAREING \& PATRICK, 1975 ; BOOTE, 1976 entre autres), on comprend que la partie aérienne ayant été modifiée par le changement de racines, ces dernières portent elles aussi la "marque " du greffon auquel elles étaient associées. Mais cette « signature » est à la fois quantitative (modification du poids sec) et qualitative : en effet la morphologie racinaire résultante est à la fois caractéristique de l'identité variétale des racines et de celle de la variété utilisée en greffon.

L'influence réciproque «quantitative " entre portegreffe et greffon chez l'aubergine rejoint les observations faites sur arbres fruitiers (TUBBS, 1951, cité par TROUGHTON \& WHITTINGTON, 1970), sur soja (CARDWELL \& POLSON, 1972 ; SUllivaN \& BRUN, 1975) et sur manioc (DAHNIYA et al., 1982). L'expérience d'ABDELHAFEEZ et al., (1975) sur des tomates greffées sur racines d'aubergine, avait mis en évidence une stimulation de la croissance végétative de la tomate (poids sec des tiges et des feuilles) mais non de la fructification (poids sec des bouquets de fruits) : mais la conduite sur une seule tige puis l'étêtage au-dessus de la $4^{\mathrm{e}}$ inflorescence suffisent à expliquer la différence des résultats. Notons que ces auteurs remarquaient que le sens et l'intensité des modifications engendrées par les racines d'aubergine variaient avec la température de l'air et du sol.

\section{CONCLUSION}

La rupture de l'unité variétale par greffage entre plantes de "Liu Yé Qié » d'origine extrême-orientale et de « LF3-24» d'origine méditerranéenne montre que la meilleure croissance végétative et le meilleur rendement s'obtiennent, dans les conditions agroclimatiques méditerranéennes, essentiellement grâce à un meilleur développement racinaire. Ce résulta! établi pour un couple variétal particulier rejoint tout à fait celui que l'on avait commencé à percevoir dans une expérience antérieure, non publiée car détrutte précocement par une attaque de stolbur, qui comparait 12 formules de greffage établies à partir de 3 variétés de chaque origine. Il sera donc intéressant de recommencer ce type d'expérience plus complète pour préciser cette fois la part «écotypique » que l'on peut imputer à l'influence réciproque entre porte-greffe et greffon. Notons seulement pour l'instant que "Liu Yé Qié » et «LF3-24 》 représentent une des distances maximum possible entre les écotypes méditerranéen et extrême-oriental (DAUNAY, 1986).

L'influence directe et positive de la vigueur $\mathrm{du}$ système racinaire sur le développement de la partie aérienne se fait très probablement grâce à un meilleur approvisionnement en eau et en éléments fertilisants. Il induit une plus grande ramification de la partie aérienne, d'où des fruits plus nombreux et plus lourds. Mais réciproquement, la vigueur de la partie aérienne se répercute sur le développement du système racinaire dont elle augmente fortement le poids sec tout en modifiant sa configuration géométrique. Parties racinaires et aériennes s'influencent donc mutuellement, mais le poids "relatif » des unes et des autres n'est équilibré que pour le nombre de fruits. En effet les racines influencent d'une façon prédominante le poids moyen et par conséquent le rendement global.

Reçu le 18 mars 1985. Accepté le 16 novembre 1985

\section{RÉFÉRENCES BIBLIOGRAPHIQUES}

Abdelhafeez A. T., Cornillon P., 1976. Effects of irrigation rhythm on growth, fruit set, yield, and quality of eggplant (Solanum melongena) in southern France. Plant and Soil, 45, 213-225.

Abdelhafeez A. T., Harssema H., Verkerk K., 1975. Effects of air temperature, soil temperature and soil moisture on growth and development of tomato itself and grafted on its own and eggplant rootstock. Sci. hortic., 3 (1), 65-73.

Beyries A., 1979. Le greffage, moyen de lutte contre les parasites telluriques des Solanées cultivées pour leurs fruits. Thèse Doct. d'Univ. Mention Sci. Nat., Univ. Sci. \& Tech. du Languedoc, Acad. Montpellier, France, $166 \mathrm{p}$

Bierhuizen J. F., 1974. Root growth and its environment. Acta hortic., 39, 127-135.

Boote K. J., 1976. Root-shoot relationships. Soil Crop Sci. Soc. of Florida, Proc., 36, 15-23.

Cardwell V. B., Polson D. E., 1972. Response of "Chippewa 64 " soybean scions to roots of different genotypes. Crop Sci., 12, 217219.

Cornillon P., Dauple P., 1981. Influence of irrigation rhythm and water supply on growth, water status and yield of eggplant (Solanum melongena L.). Plant and Soil, 59, 365-379.

Dahniya M. T., Oputa C. O., Hahn S. K., 1982. Investigating source-sink relations in cassava by reciprocal grafts. Exper. Agric., 18, 399-402.

Daunay M. C., 1982. Développement de l'aubergine et adaptation au climat: étude préliminaire. Rapp. de DEA, 22 p., Univ. AixMarseille III, Ed. Stn. Amélior. Plantes maraîchères, INRA, 84140 Montfavet, France.
Daunay M. C., 1986. Développement de l'aubergine et mécanismes d'adaptation au climat. Thèse Doct. es Sci., Univ. Aix-Marseille III (à paraître).

Filov A. I., 1958. Vegetable plants, fam. Solanaceae, Solanum melongena L., p. 292-370. In : D. D. Brezhnev : « Kul'turnay a Flora SSSR ». State agric. publ. Office, Moscow-Leningrad, $531 \mathrm{p}$

Kramer P. J., 1969. Roots and root growth, p. 105-149. In : P. J. Kramer : "Plant and soil water relationships: a modern synthesis 》. Mc Graw Hill Book Co, 473 p.

Pierre C., 1982. Greffage de l'aubergine : technique intéressante pour sols fatigués. Agrisept (883), 27-28.

Pochard E., 1979. Adaptation climatique et propriétés hydriques: réponse de l'aubergine au climat des serres. Riv. di Agron., XIII (3), 381-391.

Pochard E., Serieys H., 1974. Mise en évidence de différences variétales de transpiration chez l'aubergine et d'autres solanacées (Solanum melongena $\mathrm{L}$., Capsicum annuum L., Lycopersicon esculentum Mill.). Conséquences pour l'adaptation à différents climats. Ann. Amélior. Plantes, 24 (3), 243-268.

Sullivan T. P., Brun W. A., 1975. Effect of root genotype on shoot water relations in soybeans. Crop Sci., 15, 319-322.

Troughton A., Whittington W. J., 1970. The significance of genetic variation in root system, p. 296-313. In : W. J. Whittington. Root growih. Butterworths, London, $450 \mathrm{p}$.

Wareing P. F., Patrick J., 1975. Source-sink relations and the partition of assimilates in the plant, p. 481-500. In : J. P. Cooper : "Photosynthesis and productivity in different environments". Cambridge Univ. Press, 695 p. 